\title{
OPHERA abre a la ciudadanía las experiencias de reconstrucción de la región italiana de Las Marcas afectada por el terremoto de 2016
}

El proyecto OPHERA tiene como objetivo poner en valor, como expresión cultural, el proceso de restauración del patrimonio cultural que se está llevando a cabo en las zonas afectadas por el terremoto de 2016 en la región de Las Marcas, en el centro de Italia. Una de las premisas del proyecto, en el que participan universidades de hasta cinco países, consiste en plantear la recuperación de este patrimonio como un proceso participativo y transparente, accesible a la comunidad, a la vez que se fomenta el intercambio cultural entre profesionales europeos de la restauración y un público más amplio, compartiendo las experiencias de reconstrucción.

Giovanni Issini | Ministerio de Cultura de Italia

Pilar Montero Vilar | Dpto. de Pintura-Restauración, Universidad Complutense de Madrid

URL de la contribución <http://www.iaph.es/revistaph/index.php/revistaph/article/view/5061>

Los daños al patrimonio cultural debidos a las amenazas naturales representan una pérdida de bienes histórico-artísticos y, al mismo tiempo, una pérdida del patrimonio inmaterial, constituido por la memoria e identidad de las comunidades.

El enjambre sísmico acaecido en el centro de Italia, en la región de Las Marcas, entre agosto de 2016 y enero de 2017, afectó a un gran número de patrimonio cultural. Resultaron dañados o destruidos 1.664 iglesias históricas y 1.223 edificios protegidos (incluyendo castillos, palacios y zonas arqueológicas). Además, alrededor de 13.000 obras de patrimonio mueble con diferentes niveles de daño fueron retiradas de estos edificios y almacenadas en depósitos temporales. En cuanto al impacto paisajístico, 285 centros históricos sufrieron daños y, todavía hoy, algunos de ellos permanecen cerrados debido a las obras de consolidación y protección.

El proyecto OPHERA está formado por un consorcio de cinco asociados: el Segretariato Regionale del Ministero della Cultura de la región de Las Marcas (Italia), la Universidade do Minho, en Guimarães (Portugal), la Cyprus University of Technology en Limassol (Chipre), la Università degli Studi di Ferrara (Italia) y la Univerza $v$ Ljubljani en Liublana (Eslovenia) y tiene como obje- tivo poner en valor, como expresión cultural, el proceso de restauración del patrimonio cultural que se está llevando a cabo en las zonas afectadas por el terremoto de 2016 en la región de Las Marcas (Italia). Una de las premisas del proyecto consiste en plantear el hecho de que la recuperación de este patrimonio es un proceso a largo plazo que debe ser participativo y transparente y que, por tanto, lo disfrutarán las generaciones futuras. Los valores culturales inherentes a los procesos de restauración, que se caracterizan por una participación de profesionales procedentes de diferentes disciplinas con un rango amplio de competencias y, en algunos casos, también por la aportación creativa, generalmente solo son visibles para los círculos profesionales (arquitectos, restauradores de arte, investigadores, gestores de arte, historiadores, etc.).

El objetivo del proyecto OPHERA consiste en hacer accesible a la comunidad, en el sentido más amplio del término, todas las fases de la restauración de los casos de estudio de patrimonio cultural e histórico dañado que han sido seleccionados, a la vez que se fomenta el intercambio cultural entre profesionales europeos de la restauración y un público más amplio, compartiendo las experiencias de reconstrucción desarrolladas en las regiones del centro de Italia. 


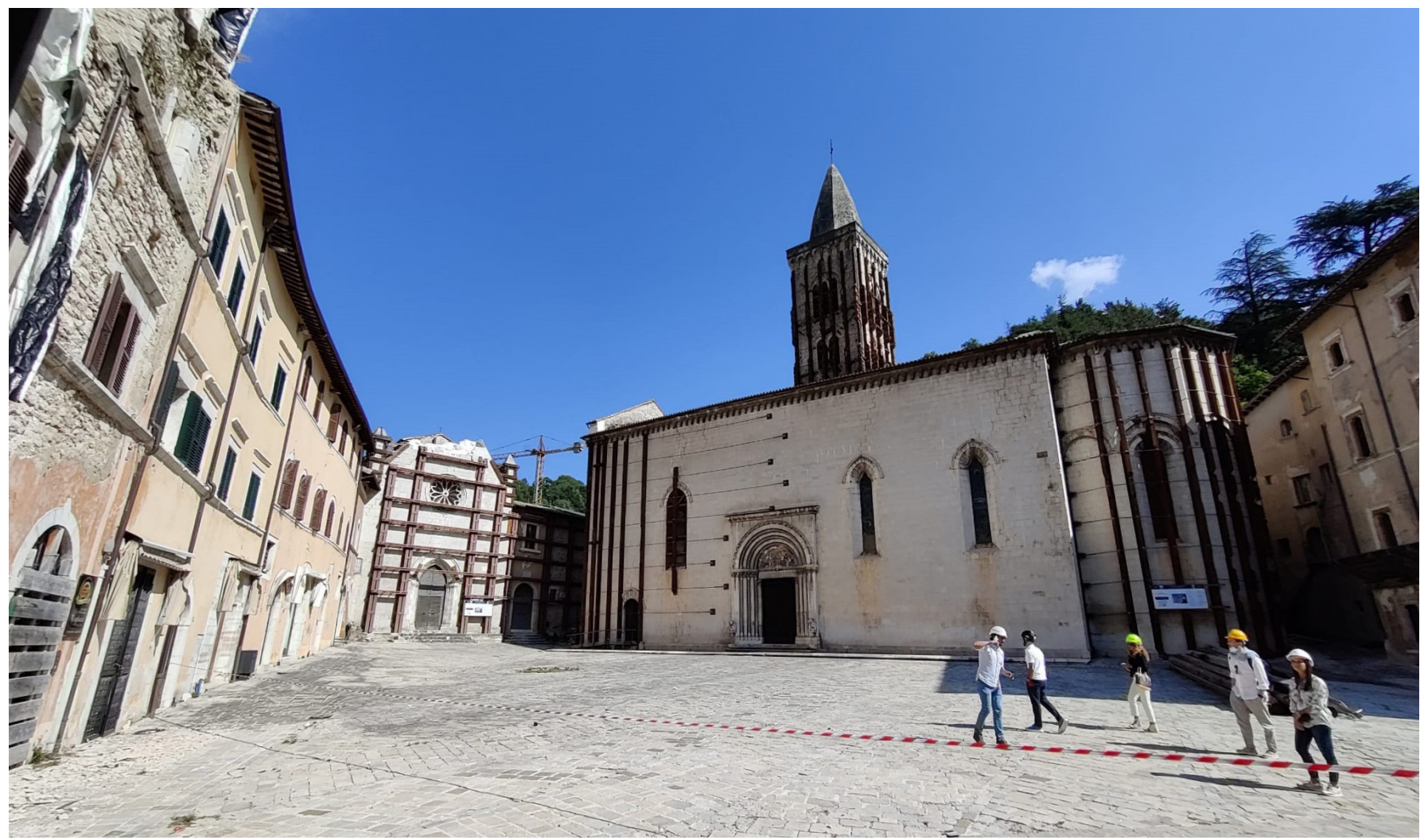

Colegiata de Santa María (s. XI-XIV) en Visso (8 de septiembre de 2021). Tercer taller celebrado en Camerino, 6-10 de septiembre de 2021 | foto Alessia Travanti

El proyecto, financiado por el programa Europa Creativa de la Unión Europea, diseñó una convocatoria pública a nivel europeo en la que fueron seleccionados 33 participantes con formación en diferentes campos (arquitectura, ingeniería, conservación-restauración, historia del arte, geología, gestión de patrimonio cultural, publicidad...) de 7 países de Europa que conformaron dos equipos, el $\mathrm{A}$ y el $\mathrm{B}$.

En la primera fase del proyecto se celebraron tres talleres.

El primero de ellos, en el que participaba el equipo A, estaba organizado por la Cyprus University of Technology, el Digital Heritage Research LAB (DHRLab), la Cátedra UNESCO de Patrimonio Cultural Digital y la Cátedra ERA Mnemosyne. Aunque estaba previsto que se celebrase de forma presencial y el equipo $A$ acudiese a Limassol (Chipre), debido a la COVID-19 finalmente se celebró de forma online, entre el 17 y el 21 mayo de
2021. Entre los objetivos del taller se encontraba proporcionar a los participantes una visión general de los métodos, tecnologías y herramientas digitales disponibles aplicadas al conocimiento y la comunicación del patrimonio cultural, especialmente a los objetos en proceso de restauración en el escenario posterremoto; y promover la formación sobre el método de comunicación y visualización de datos técnicos complejos para llegar y sensibilizar a un público más amplio.

El taller consistió en una serie de conferencias online y en la realización de un trabajo personal de cada una de las personas participantes seleccionadas a partir de alguno de los presupuestos del taller y en una puesta en común de los resultados.

El segundo de los talleres, celebrado presencialmente en Guimarães (Portugal), entre el 28 de junio y el 1 de julio del mismo año, y al que acudió el equipo $B$, estuvo organizado por la Universidad de Minho y el Instituto 


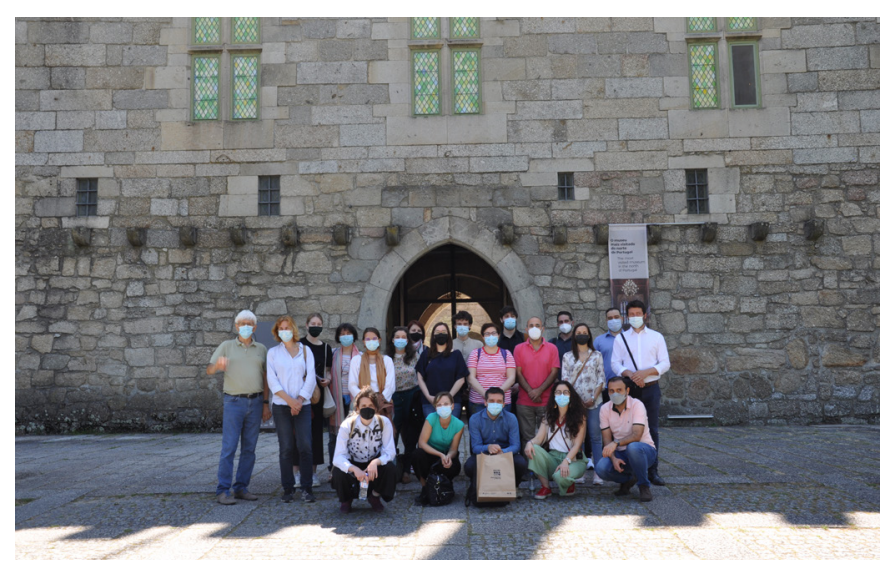

Segundo taller realizado en Guimarães. 28 de junio-2 de julio de 2021. El grupo de OPHERA delante del Palacio Ducal de Guimarães | foto OPHERA Project

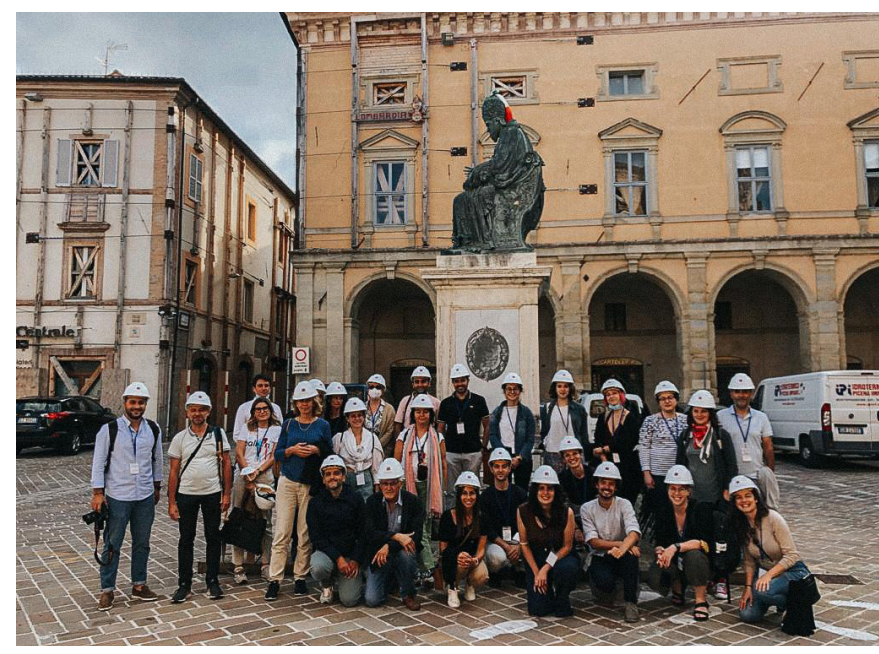

Tercer taller. Plaza de la catedral, Camerino, 6 de septiembre de 2021. Participantes proyecto OPHERA | foto OPHERA Project

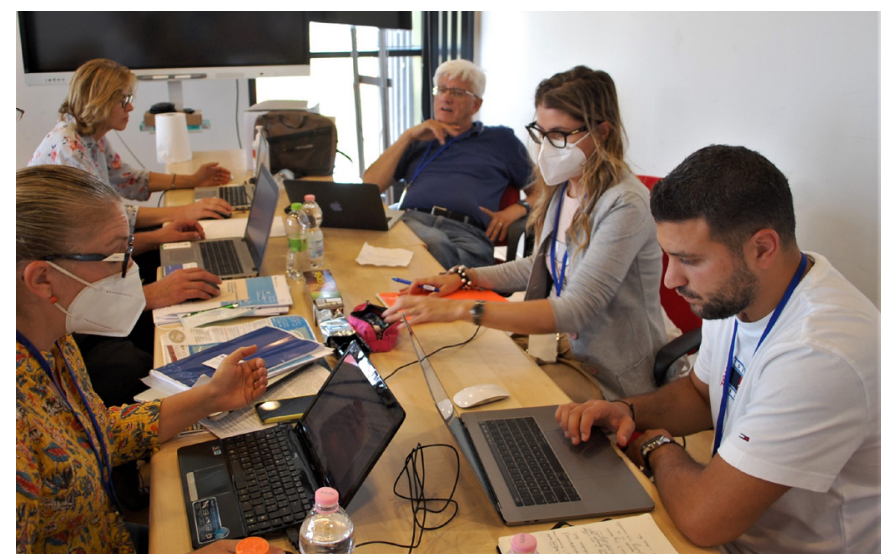

Taller en Camerino, Italia. 6-10 de septiembre de 2021 | foto Maurizio Bilò para la Sostenibilidad y la Innovación en la Ingeniería Estructural (ISISE), centro de investigación de I+D de la Universidad de Coimbra y la Universidad de Minho. En él tuvieron la oportunidad de abordar el estudio de los métodos tradicionales e innovadores para la supervisión estructural y la evaluación de la seguridad del patrimonio cultural construido. De nuevo se impartieron conferencias, se realizaron ejercicios prácticos sobre análisis e interpretación de datos y sistemas de monitorización y aplicaciones de laboratorio, así como la visita a uno de los caso de estudio: el Palacio Ducal de Guimarães.

Entre los objetivos de este segundo taller se encontraban proporcionar a los participantes una visión general de las técnicas tradicionales y avanzadas de control estructural y evaluación de la seguridad del patrimonio arquitectónico; formar a los profesionales sobre las mejores prácticas en el ámbito de la supervisión de la salud estructural sísmica y la conservación preventiva; y aumentar la concienciación de los operadores culturales sobre la importancia de las inspecciones periódicas y las prácticas de supervisión del patrimonio cultural construido, así como sobre los beneficios sociales y económicos asociados a las acciones de prevención.

Finalmente, tanto el equipo A como el B participaron en el tercer taller que estaba organizado por la Universidad de Ferrara y el Ministero della Cultura de Las Marcas y que tuvo lugar en Camerino (Italia) entre el 6 y el 10 de septiembre de 2021.

Camerino es uno de los pueblos más severamente afectados por los eventos del terremoto de 2016 y que, a día de hoy, como se pudo comprobar in situ, continúa con su casco histórico cerrado. En esta ocasión el objetivo consistía en definir el contenido cultural específico del proceso de restauración que se mostrará durante las jornadas de puertas abiertas que se celebrará en la segunda fase del proyecto y adquirir los datos necesarios para realizar dichos contenidos. El taller permitió formar a los participantes en el uso de métodos y herramientas avanzadas para la monitorización, el levantamiento digital y la comunicación del patrimonio cultural construido en proceso de restauración, que también se aplicarán a las actividades de reconstrucción posteriores. 
Los participantes formaron 6 equipos, de 3-4 participantes más 2 tutores y a cada uno de ellos se les asignó un lugar de trabajo específico. Estos lugares clave de la restauración en curso, situados en las ciudades más afectadas de la región de Las Marcas, fueron la Colegiata de Santa María (s. XI-XIV) y el Palazzo del Prior (s. XIII$\mathrm{XVI}$ ) en Visso, el Palacio Saladini di Rovetino (s. XVII) en Ascoli Piceno, el Castillo di Luco (s. XI-XIII), el complejo de Sant'Agostino en Pieve Torina y, por último, el Palazzetto Urbani (s. XIV) y la Casa Campili (s. XIII) en Monte San Martino. Cada uno de los equipos tuvo la oportunidad de visitar el lugar asignado acompañado de las autoridades locales, arquitectos, restauradores y propietarios y realizó un trabajo de campo consistente en la recogida de opiniones y aspiraciones de las comunidades locales sobre la restauración y reconstrucción de su propio patrimonio cultural y en diseñar las actividades que se realizarán en las jornadas de puertas abiertas este año 2022.

La segunda fase del proyecto consiste en la celebración de unas jornadas de puertas abiertas con las que se pretende fomentar un intercambio cultural entre los profesionales de la restauración y un público más amplio representado por ciudadanos, turistas, administradores locales, estudiantes y asociaciones culturales, con el fin de transformar el proceso de restauración en un acontecimiento cultural, que recoja el espíritu de la Convención de Faro (2005) en relación con la participación de toda la sociedad en la gestión del patrimonio cultural, entendido como recurso para el desarrollo sostenible y la calidad de vida de una sociedad en continua evolución.

Los equipos participarán en la organización de las dos jornadas de puertas abiertas de los seis lugares seleccionados. Estas jornadas consistirán en la puesta en práctica de las actividades diseñadas en el tercero de los talleres realizado en Camerino en septiembre de 2021 y se celebrarán los fines de semana del 28 y 29 de mayo y el 4 y 5 de junio de 2022 . 